\title{
The Removal of Airborne Molecular Contamination in Cleanroom Using PTFE and Chemical Filters
}

\author{
Ching-Fa Yeh, Chih-Wen Hsiao, Shiuan-Jeng Lin, Chih-Min Hsieh, Toshio Kusumi, Hideki Aomi, Hideo Kaneko, \\ Bau-Tong Dai, and Ming-Shih Tsai
}

\begin{abstract}
Cleanroom contamination and its impact on the performance of devices are beginning to be investigated due to the increasing sensitivity of the semiconductor manufacturing process to airborne molecular contamination (AMC). A clean bench was equipped with different filter modules and then most AMC in the cleanroom and in the clean bench was detected through air-sampling and wafer-sampling experiments. Additionally, the effect of AMC on device performance was examined by electrical characterization. A combination of the NEUROFINE PTFE filter and chemical filters was found to control metal, organic, and inorganic contamination. We believe that the new combination of filters can be used to improve the manufacturing environment of devices, which are being continuously shrunk to the nanometer scale.
\end{abstract}

Index Terms-Airborne molecular contamination, chemical filter, glass fiber filter, PTFE filter.

\section{INTRODUCTION}

$\mathbf{T}$ HE cleanness of a cleanroom environment must be tightly controlled to achieve high-yield and high-performance ULSI manufacturing [1]-[4]. Recently, new developments of material and process have helped to shrink device geometry. The advanced contamination control of cleanrooms has also been indispensable in this regard [5]. So far, only particle contamination has been intensively studied [6], and recently developed filters, such as HEPA and ULPA, can already sufficiently suppress particles [7]. However, the controllability of airborne molecular contamination (AMC) by present cleanroom technologies has not yet been validated because AMC is a kind of atom or molecular-level gas-phase contamination like organic and inorganic ones. As the minimum feature size of devices is continuously scaled down to far below $0.1 \mu \mathrm{m}$, AMC will gradually become crucial in ULSI manufacturing. In the near future, especially in the nanodevice era, contamination by organic compounds, inorganic ions, and trace doping impurities [8] in cleanroom air may also dominate the characteristics, reliability, and even yield of devices. Technologies for eliminating both particles and AMC from manufacturing environment must be considered to completely solve this problem.

Manuscript received January 21, 2003; revised November 13, 2003

C.-F. Yeh, C.-W. Hsiao, S.-J. Lin, and C.-M. Hsieh are with the Department of Electronics Engineering and Institute of Electronics, National Chiao-Tung University, Hsinchu, Taiwan, R.O.C. (e-mail: yehcf@beige.plala.or.tw; cwhsiao.ee87g@nctu.edu.tw; sjlinf@tsmc.com).

T. Kusumi, H. Aomi, and H. Kaneko are with Daikin Industries, Ltd., Osaka 566-8585, Japan.

B.-T. Dai and M.-S. Tsai are with the National Nano Device Laboratories, Hsinchu, Taiwan, R.O.C. (e-mail: btdai@ndl.gov.tw; mstsai@ndl.gov.tw). Digital Object Identifier 10.1109/TSM.2004.826957

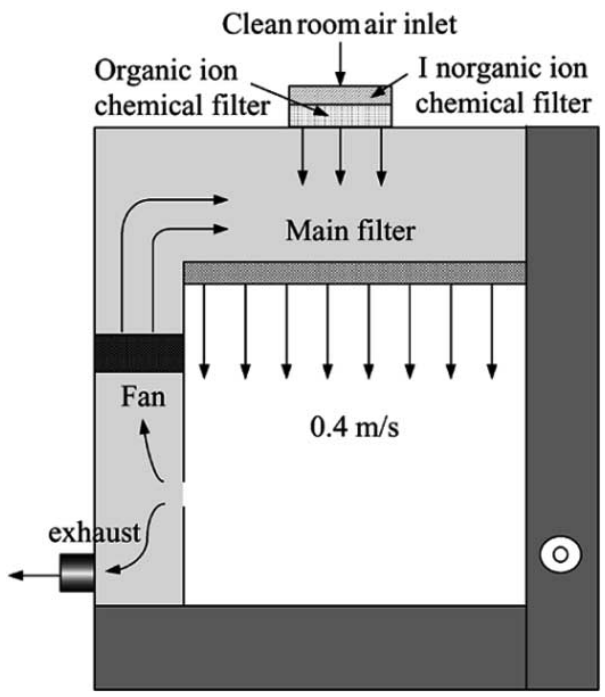

Fig. 1. Schematic diagram of specially designed clean bench.

To date, several investigations of AMC and its effects on device performance have been conducted. Most, however, have only paid attention to the experiments based on intentional contamination on devices [3], [9], [10]. Few studies have considered AMC absorbed from cleanroom air and its practical effects on the characteristics of devices.

In this paper, a clean bench was specially equipped with different filter modules such as the NEUROFINE polytetrafluoroethylene (PTFE) filter, the glass fiber ULPA filter, and a combination of chemical filters with both of these. All elements in the air or on the wafer were analyzed through air sampling and wafer sampling inside the bench, to determine clearly the ability of each filter module to suppress AMC. The practical effects of AMC on device performance were also investigated by actually exposing a wafer to air in an especially controlled clean bench before gate oxidation.

\section{EXPERIMENT}

A specially controlled and fabricated clean bench (CB) was set up in the cleanroom (CR) of a class $10 \mathrm{k}$ in a laboratory. Fig. 1 schematically depicts the $\mathrm{CB}$. Cleanroom air, from the air inlet of the $\mathrm{CB}$, was initially filtered with the filter module that included inorganic and organic ion chemical filters, before flowing through the main filter, which was designed to be changeable. The chemical filter is mainly composed of active carbon, and it can absorb ions. Two kinds of main filter were used, the NEUROFINE PTFE filter and the glass fiber ULPA 


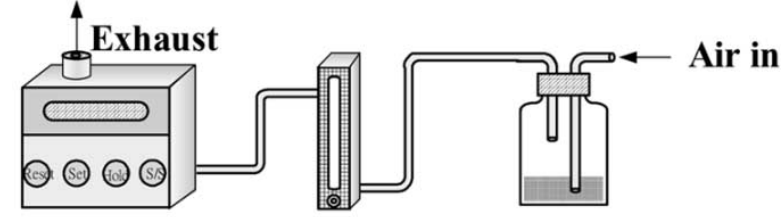

Pump Flow Meter

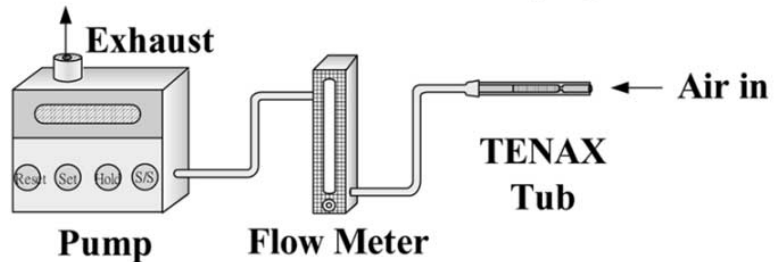

Fig. 2. Air-sampling equipments used for evaluation of metals, inorganic, and organic contaminations.

filter (GF). The air-flow rate and turnover rate inside the $\mathrm{CB}$ were set to $0.4 \mathrm{~m} / \mathrm{s}$ and $5 \%$, respectively. Before sampling, the CB was first operated for more than one week to stabilize environmental conditions.

\section{A. Evaluation of AMC Through Air Sampling}

Fig. 2 presents the air-sampling tools, Impinger and TENAX, which were equipped with a flow meter and a pump. The flow rate and sampling time of Impinger were $2 \mathrm{~L} / \mathrm{min}$ and $24-72 \mathrm{~h}$, while those of TENAX were $100 \mathrm{~mL} / \mathrm{min}$ and $5 \mathrm{~h}$. Impingers collected mainly both inorganic and metallic contamination elements. The former were analyzed by the ion chromatography (IC), while the latter were analyzed using an inductively coupled plasma mass spectrometer (ICP-MS). Organic substances were collected by a TENAX (a kind of absorbent) tube and quantitatively analyzed by a gas chromatograph mass spectrometer (GC-MS). The air in the CR was also analyzed for comparison. In all sampling results, one standard deviation was defined as the error range.

\section{B. Evaluation of AMC Through Wafer Sampling}

The AMC absorbed on the surfaces of actual 6-in P-type (100) $\mathrm{Si}$ wafers was analyzed for each filter module. After standard Radio Corporation of America (RCA) cleaning, wafers were divided into two parts and exposed to the air in the CR and the CB for 24-72 $\mathrm{h}$. The metal contamination on these wafer surfaces was analyzed using total reflection $\mathrm{X}$-ray fluorescence (TRXRF), while the organic contamination was analyzed by thermal desorption system, atmospheric pressure ionized mass spectrum (TDS-APIMS). For comparison, the contamination of a wafer not exposed to any environment was evaluated as a control.

\section{Evaluation of AMC Through Electrical Characterization}

Metal oxide semiconductor (MOS) capacitors, with thermal oxide as the insulator, were prepared to study the effects of AMC on practical device characteristics. Fig. 3 shows the process flow diagram for preparing a MOS capacitor. The active region was first defined on a 6-in P-type (100) Si wafer by local oxidation of the silicon (LOCOS) process, and then a 40-nm-thick sacrificial

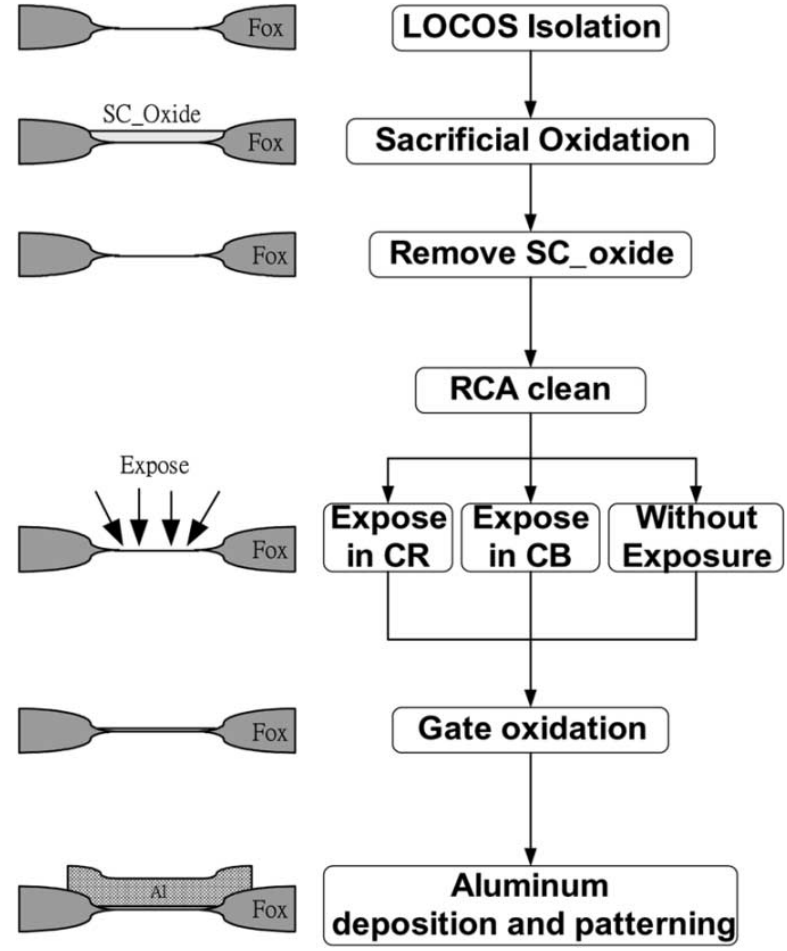

Fig. 3. Process flow of MOS capacitor exposure experiment.

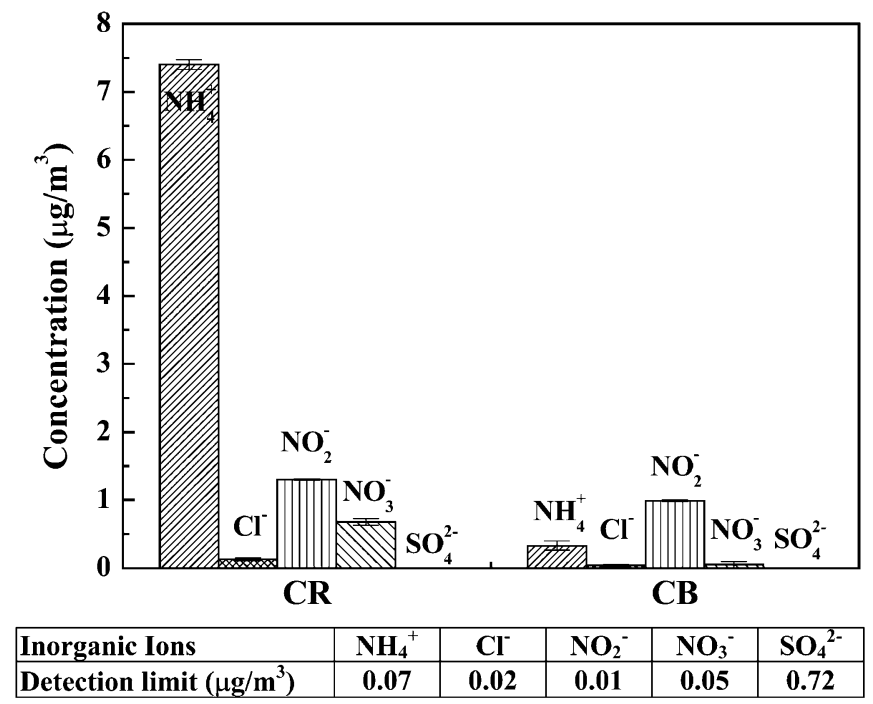

Fig. 4. Air-sampling results of inorganic ions.

oxide layer was formed by thermal oxidation. This sacrificial oxide was subsequently removed, and the wafers were further cleaned by RCA cleaning before exposure. After exposure in the $\mathrm{CR}$ or the $\mathrm{CB}$ for $4 \mathrm{~h}$, a 5 -nm-thick gate oxide layer was grown on each wafer by thermal oxidation, and finally an aluminum gate electrode was deposited and patterned. Unexposed blank samples were also prepared using the same procedure. Finally, for all samples, the electrical properties such as leakage current density versus electrical field (J-E), breakdown field $\left(\mathrm{E}_{\mathrm{bd}}\right)$, and charge to breakdown $\left(\mathrm{Q}_{\mathrm{bd}}\right)$ were measured through the MOS capacitor $(200 \mu \mathrm{m} \times 200 \mu \mathrm{m})$ using the HP4156 semiconductor parameter analyzer. 


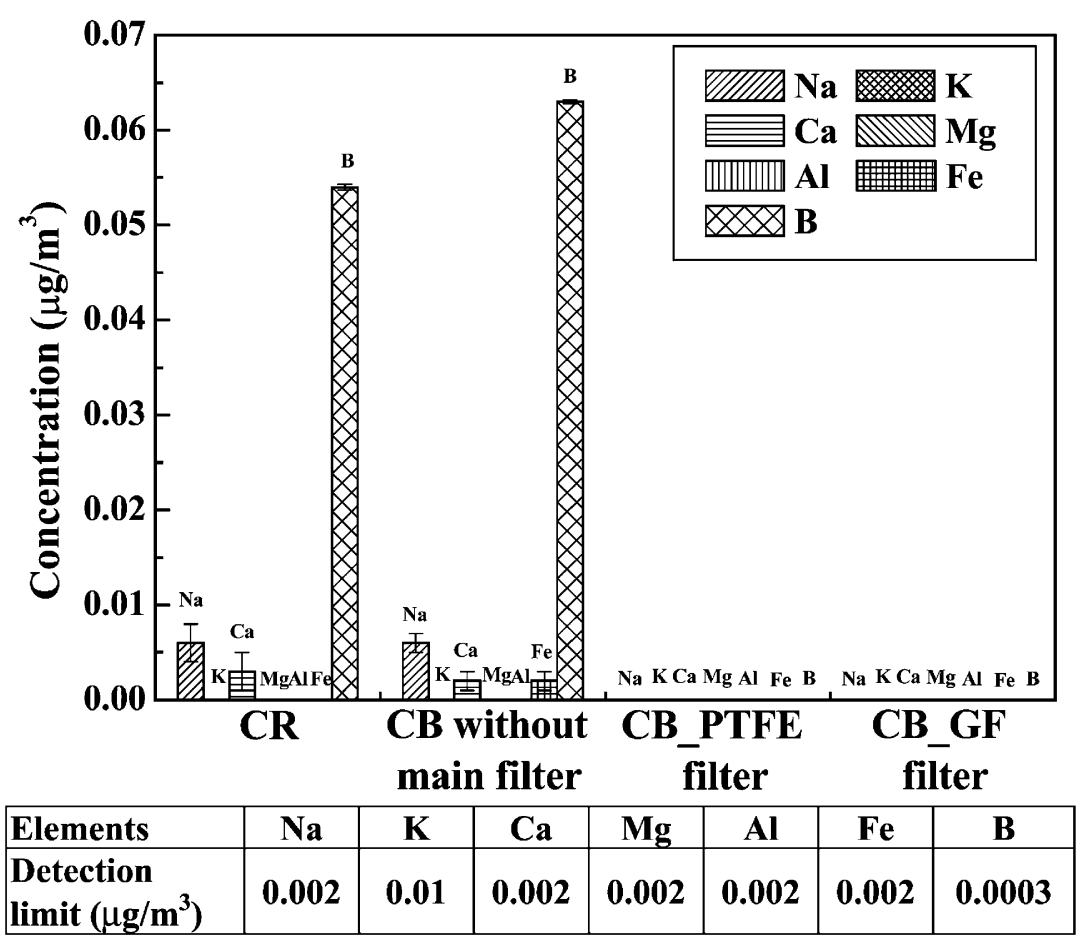

Fig. 5. Air-sampling results of metals and boron.

\section{RESULTS AND DisCUSSION}

\section{A. Air Sampling}

First, the constituents of the air in the $\mathrm{CR}$ and the $\mathrm{CB}$, equipped with only inlet chemical filters but no main filter, were evaluated. Fig. 4 shows the IC results for inorganic ion concentrations of the air in the $\mathrm{CR}$ and the $\mathrm{CB}$. The concentrations of inorganic ions such as $\mathrm{NH}_{4}^{+}, \mathrm{Cl}^{-}, \mathrm{NO}_{2}^{-}, \mathrm{NO}_{3}^{-}$, and $\mathrm{SO}_{4}^{2-}$ were found to be high in the CR. In contrast, the inorganic ions concentrations in the $\mathrm{CB}$ were obviously low, implying that the $\mathrm{CB}$ without a main filter but with chemical filters can still remove the inorganic contaminations, especially $\mathrm{NH}_{4}^{+}$ions. Next, the PTFE filter was installed as the main filter in the $\mathrm{CB}$ and the same sampling procedure was implemented. The result was very similar to that obtained only with the chemical filters. These experimental results suggest that chemical filters efficiently reduced inorganic ion contaminations [11].

Fig. 5 presents the ICP-MS results of metals and boron concentrations in the air from the $\mathrm{CR}$ and the $\mathrm{CB}$. The concentrations of $\mathrm{K}, \mathrm{Mg}$, and $\mathrm{Al}$ elements were all below the detection limit in the air from the $\mathrm{CR}$ and from the $\mathrm{CB}$ without main filter, but the concentrations of $\mathrm{Na}, \mathrm{Ca}$, and especially $\mathrm{B}$ were detected. However, for the CB with either PTFE or GF as the main filter, both the metal and the boron contamination levels were under the detection limit. In fact, the chemical filters do not remove boron or metal contamination. Boron and metal contamination can be reasonably considered to exist in complexes with particles and are thus more efficiently eliminated when the main filter is used than when it is not.

The concentrations of organic compounds in the air from the $\mathrm{CR}$ and the $\mathrm{CB}$ were also compared. As shown in Fig. 6, the organic compounds can be mainly divided into Si-based and oil-based ones. The former included D3 $\left(\mathrm{C}_{6} \mathrm{H}_{18} \mathrm{O}_{3} \mathrm{Si}_{3}\right)$,

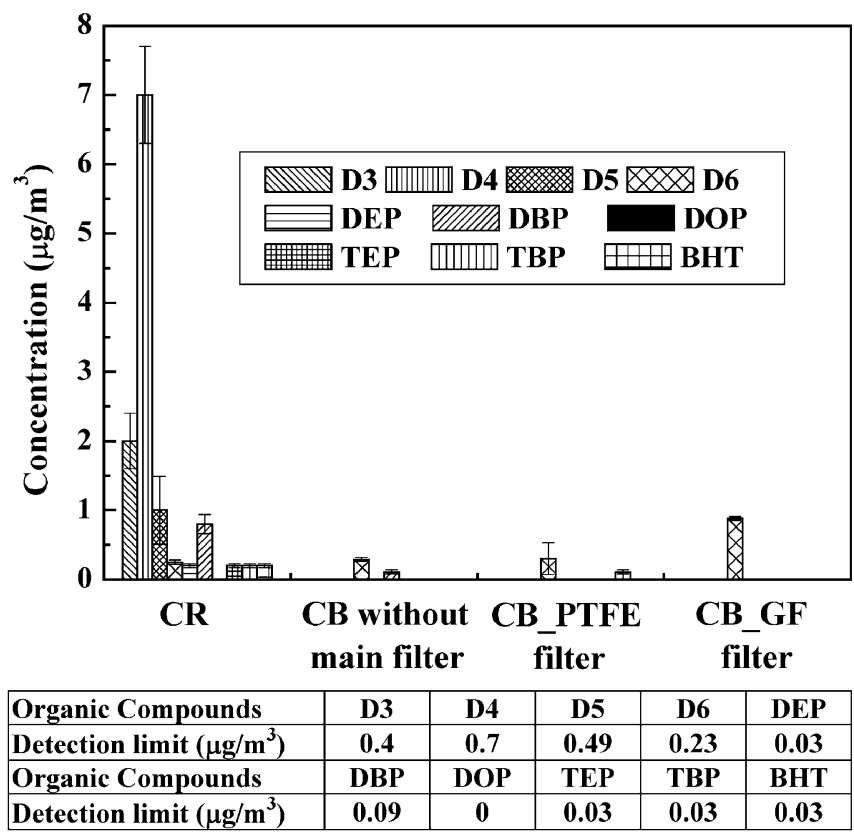

Fig. 6. Air-sampling results of organic compounds.

D4 $\left(\mathrm{C}_{6} \mathrm{H}_{24} \mathrm{O}_{4} \mathrm{Si}_{4}\right)$, D5 $\left(\mathrm{C}_{10} \mathrm{H}_{30} \mathrm{O}_{5} \mathrm{Si}_{5}\right)$, and D6 $\left(\mathrm{C}_{12} \mathrm{H}_{36} \mathrm{O}_{6} \mathrm{Si}_{6}\right)$, while the latter included DEP $\left(\mathrm{C}_{12} \mathrm{H}_{14} \mathrm{O}_{4}\right)$, DBP $\left(\mathrm{C}_{16} \mathrm{H}_{22} \mathrm{O}_{4}\right)$, DOP $\left(\mathrm{C}_{24} \mathrm{H}_{38} \mathrm{O}_{4}\right)$, TEP $\left(\mathrm{C}_{8} \mathrm{H}_{15} \mathrm{O}_{4} \mathrm{P}\right)$, TBP $\left(\mathrm{C}_{12} \mathrm{H}_{27} \mathrm{O}_{4} \mathrm{P}\right)$, and BHT $\left(\mathrm{C}_{15} \mathrm{H}_{24} \mathrm{O}\right)$. The $\mathrm{CR}$ air included high quantities of both kinds of organic compounds, but they can be mostly removed when only chemical filters were used. Of course, the concentrations can be further reduced using a combination of chemical filters and the PTFE or the GF main filter. Notably, however, a high concentration of D6 remains in the CB with a GF filter, perhaps because this compound is present in the binder of the 


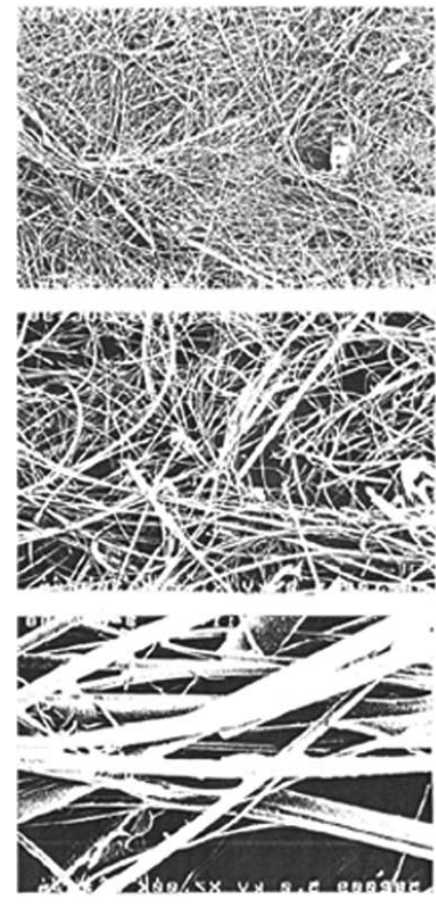

(a)
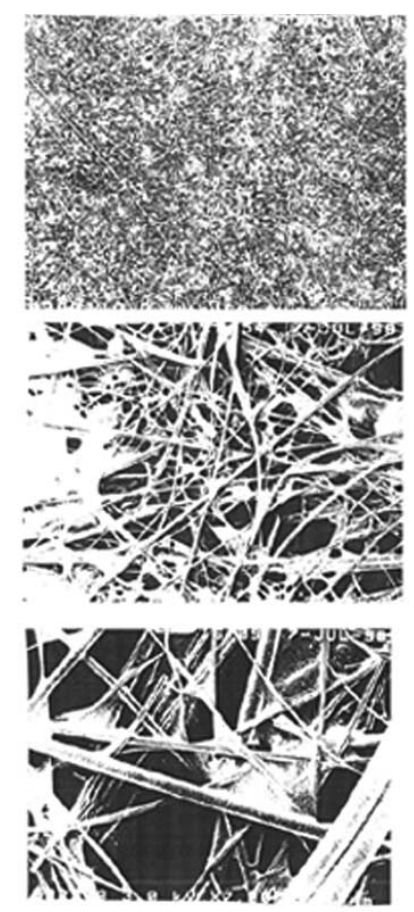

(b)
Fig. 7. Fiber structure of (a) PTFE fiber and (b) glass fiber.

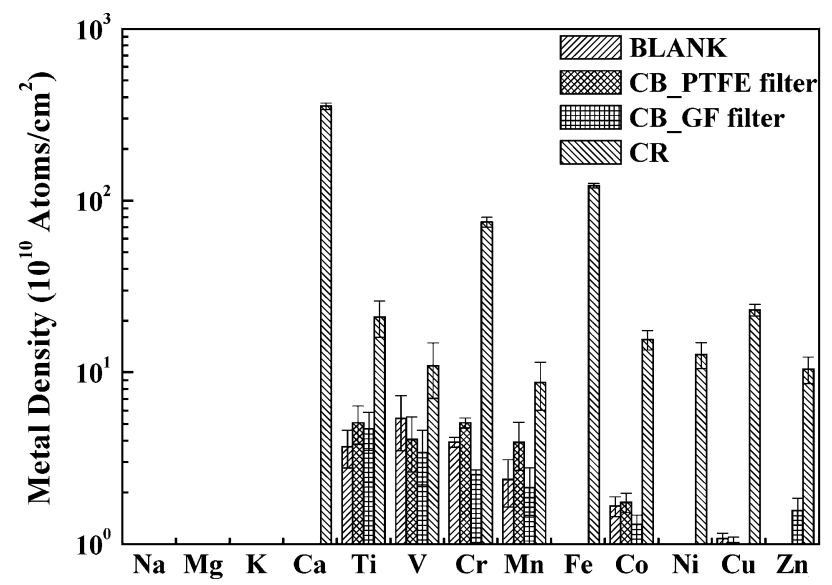

Fig. 8. Wafer sampling results of metals.

glass fiber ULPA filter or in some of the materials used to manufacture the filter. Fig. 7 shows the essential difference between the fiber structure of the PTFE filter and that of the GF filter. The glass fiber used as an intermediate in the GF filter is usually shorter than the PTFE fiber used in the PTFE filter. Hence, it must be mixed with a binder to reduce the gaps and ensure that the filter can isolate particles. However, the binder, which consists mainly of acrylic, is an organic contamination source of D6. Consequently, the total concentration of organic compounds present when a GF filter is used exceeds that obtained when a PTFE filter is used. The high organic contamination may degrade the characteristics of devices.

\section{B. Wafer Sampling}

Metal contamination on wafers exposed in the $\mathrm{CR}$ or the $\mathrm{CB}$ was quantified by TRXRF analysis. As shown in Fig. 8, the densities of metal on different wafers are compared. The "BLANK" sample refers to a wafer treated only by RCA cleaning and unexposed to air before analysis. On each wafer, five points were analyzed and the averages of the results at these points were compared. The metal densities on the BLANK and the CB samples were in the order of $10^{10}$ atoms $/ \mathrm{cm}^{2}$, but were $10^{12}$ atoms $/ \mathrm{cm}^{2}$ on the $\mathrm{CR}$ samples, again showing that the $\mathrm{CB}$ sample, when a main filter(PTFE or GF) is used, has the same concentration of metal as the BLANK sample. The results of TRXRF are rather consistent with those obtained by ICP-MS analysis, shown in Fig. 5. In PTFE or GF filter environments, the metal concentrations are the same, so the PTFE filter can replace the commercial glass fiber ULPA filter to meet the requirements of an advanced cleanroom.

Next, the intensity of organic contaminant species on the wafer surfaces, exposed or unexposed to air, were evaluated using TDS-APIMS. Fig. 9 compares results for different samples. The concentrations of each specifies of organic contamination of the CR sample greatly exceeded that of the CB sample, revealing that the wafer surface exposed in the CR does absorb much organic contamination. Here, mass numbers $m / z=16,45,73,99,149$ were chosen. Contamination peaks at $m / z=16,45,149$ often come from the outgassing of oil based plasticizers, which are widely used in plastic products of the cleanroom, such as DEP, DBP, and DOP. Peaks at $m / z=73,99$ are often associated with the absorption of Si-based siloxane, such as D5 and D6. For all the CB samples, the intensity of $m / z=99$ for a GF filter exceeds that for a PTFE filter. The result is quite consistent with the air-sampling results presented in Fig. 6. Overall, a combination of chemical and PTFE filters in a CB can provide an excellent environment with rather low concentrations of organic compounds for manufacturing advanced ULSI devices.

\section{Electrical Characterization Using an MOS Capacitor}

Fig. 10 compares J-E curves of the samples exposed in the CB and the BLANK sample. Fig. 10(a) reveals that the sample exposed under a PTFE filter has a similar leakage current density and breakdown field to the BLANK sample. The environment in the CB with the PTFE filter is excellent so the sample exposed even for $24 \mathrm{~h}$ shows little degradation. On the contrary, as shown in Fig. 10(b), the sample under a GF filter reveals a large leakage current density and exhibits much earlier breakdown than the BLANK sample. Fig. 11 compares the distributions of the breakdown electrical fields for these three kinds of samples. The breakdown fields were 13-14 MV/cm for the BLANK samples, while they were spread over a wide range, but still mainly $12-13 \mathrm{MV} / \mathrm{cm}$, for the PTFE samples. However, for the GF samples, the distribution was spread even more widely and shifted to a low electrical field. The degradation of the leakage current and the breakdown field can be attributed to AMC, which, when adhering to the wafer surface may induce defects within the gate oxide. While the MOS device is operating, the intermediate oxide defects will result in electron tunneling, rapidly inducing more defects until defect clusters form a conductive path between the substrate and the gate.

Fig. 12 plots the cumulative probabilities of leakage current densities for these three kinds of samples. In each case, the leakage current density was measured at $4 \mathrm{MV} / \mathrm{cm}$. The PTFE 


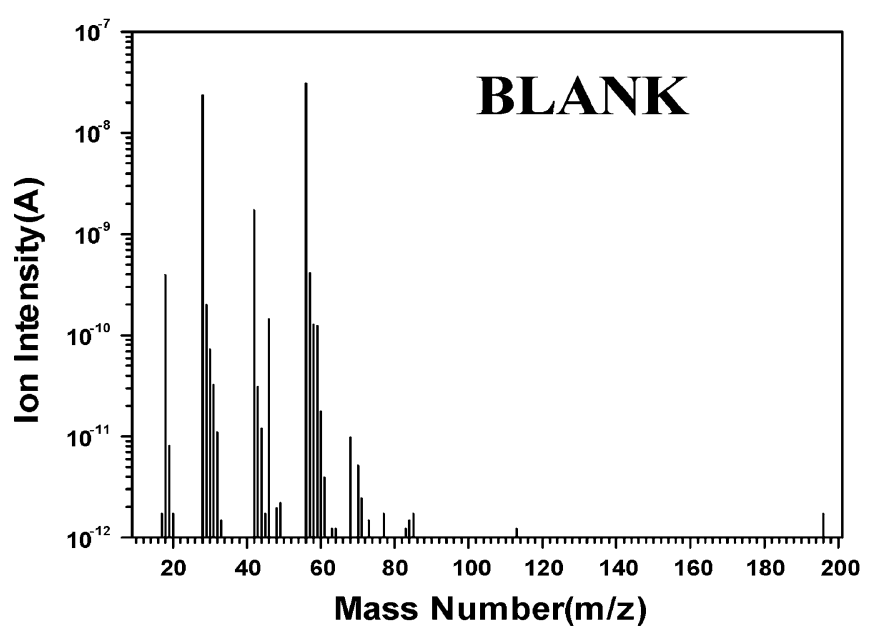

(a)

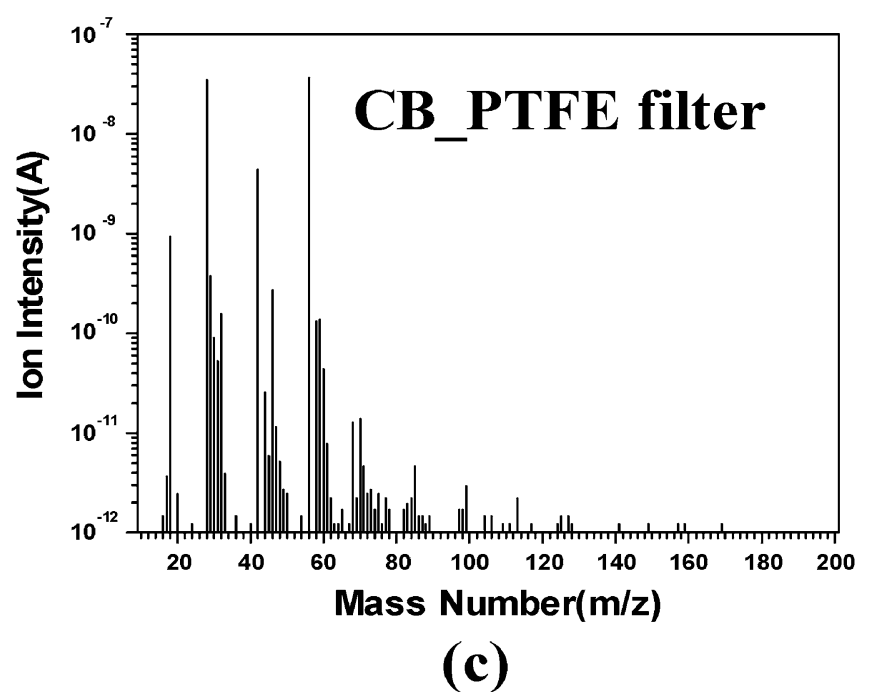

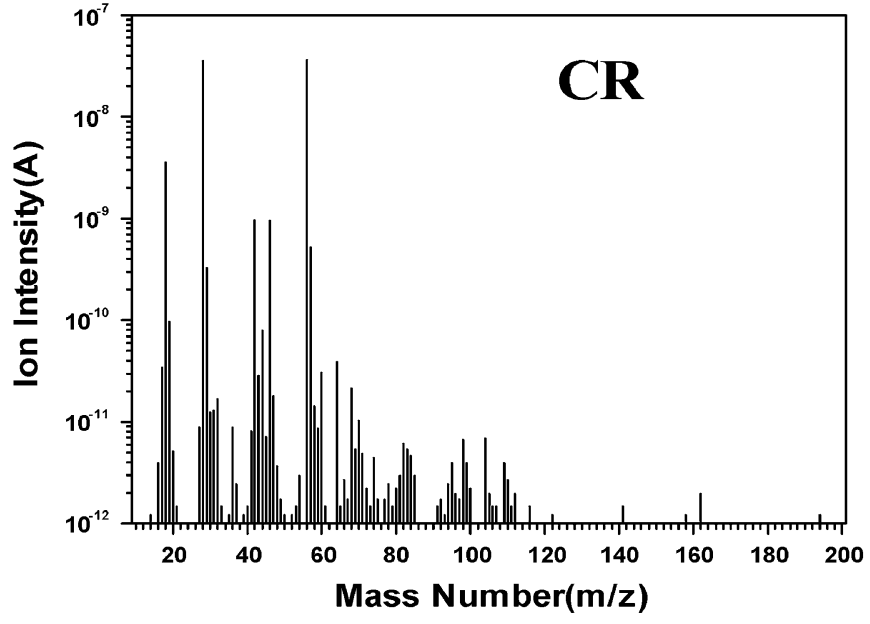

(b)

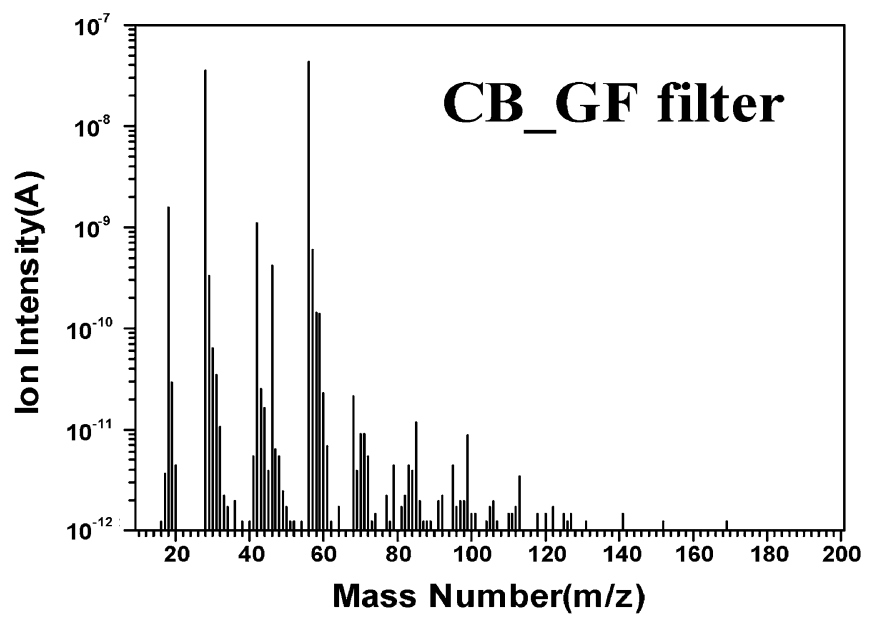

(d)

Fig. 9. Wafer sampling results of organic compounds: (a) blank wafer, (b) cleanroom, (c) PTFE filter, and (d) glass fiber filter.

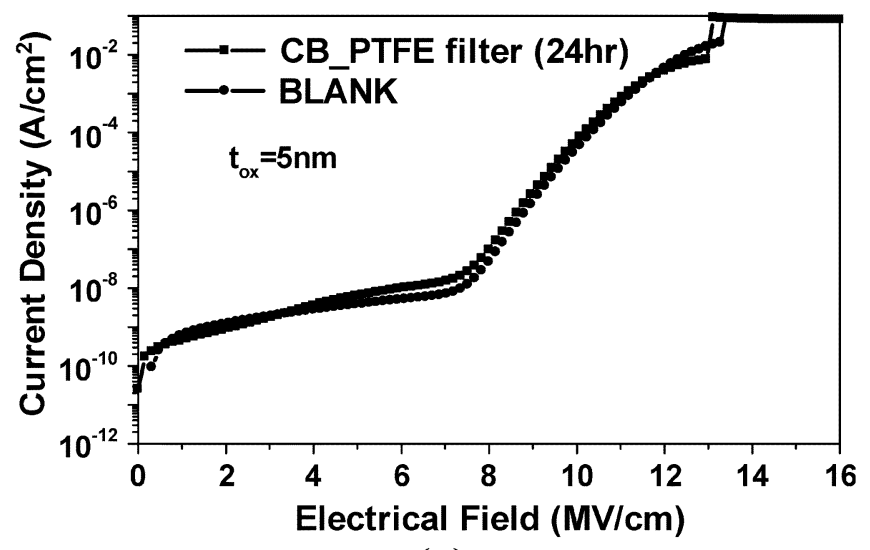

(a)

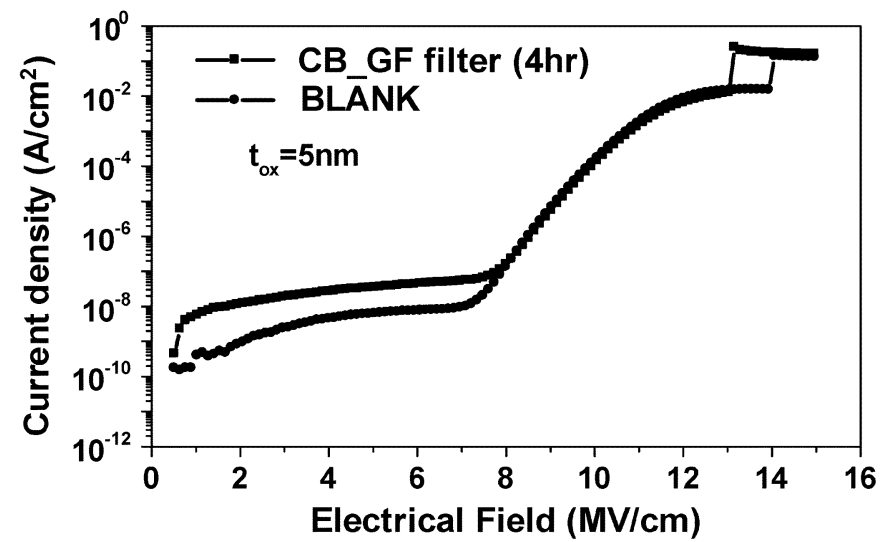

(b)

Fig. 10. J-E curves of MOS capacitors: (a) PTFE filter and (b) glass fiber filter.

samples perform similarly to the BLANK samples because the CB with the PTFE filter can create an excellent environment. In contrast, the GF samples still show a higher and less uniform leakage current density distribution than the PTFE and the BLANK samples. Fig. 13 displays a Weibull plot of charge to breakdown $\left(Q_{\mathrm{bd}}\right)$ for different samples. The PTFE samples showed slight degradation in $Q_{\text {bd }}$ compared to the BLANK samples, but the GF samples exhibited obvious degradation with low and nonuniform distribution. The above electrical results indicate that the PTFE filter is much better than the GF filter at 


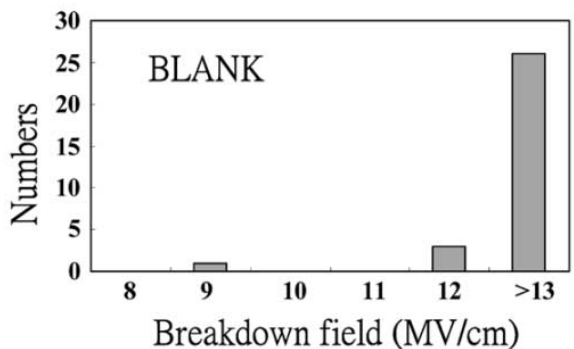

(a)

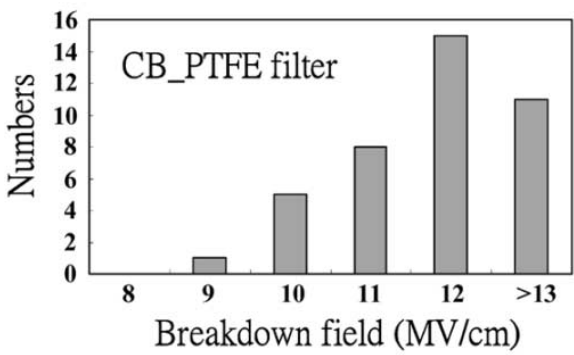

(b)

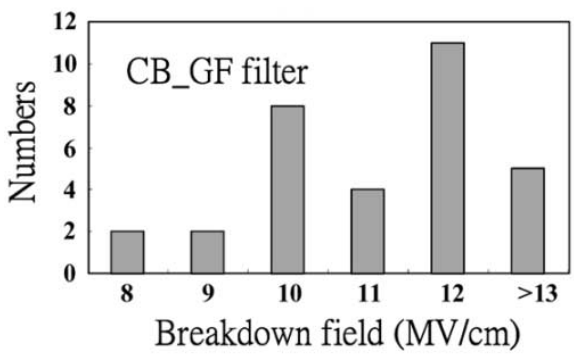

(c)

Fig. 11. Breakdown field distribution of MOS capacitors: (a) blank wafer, (b) PTFE filter, and (c) glass fiber filter.

creating an advanced clean environment for manufacturing devices.

\section{CONCLUSION}

AMC in air and on the surfaces of wafers was quantitatively analyzed. Air-sampling results reveal that a CB with chemical filters can only effectively remove inorganic ions, while one further equipped with a PTFE main filter can eliminate not only organic contaminations but also boron and metal. A CB with a GF filter still has a high concentration of D6. Wafer sampling results also demonstrate that each filter can, indeed, reduce the metal concentration to a level that corresponds to no exposure to air. Wafers exposed under a PTFE filter have less organic contaminations than those in the CR, but a GF filter shows high contamination of silicon-based organic D6 because of the binder used in the GF filter. In summary, the results obtained by air and wafer sampling are highly consistent.

The effects of AMC on device performance were also investigated using an MOS capacitor. The samples exposed in the CB under the PTFE filter have nearly the same leakage current density and breakdown field distribution as the BLANK sample, implying that the exposed wafers have few defects and exhibit slight degradation of film quality. However, the samples in the CB under a GF filter suffer an obviously degraded breakdown

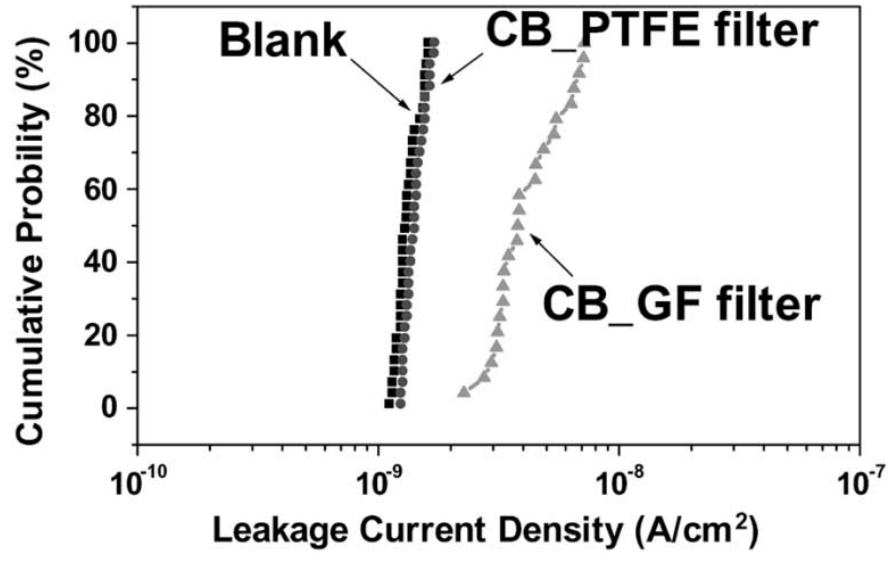

Fig. 12. Cumulative probability of leakage current density under different exposure environments.

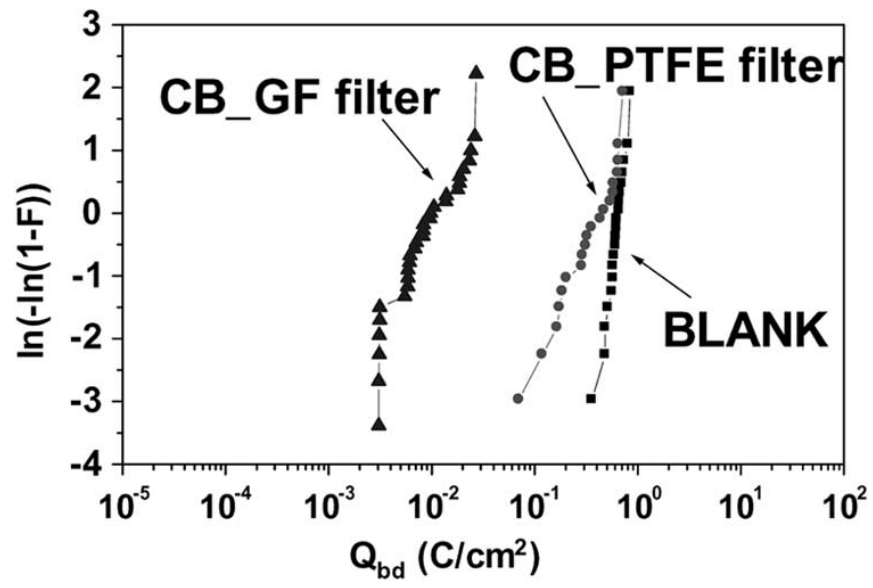

Fig. 13. $Q_{\mathrm{bd}}$ Weibull plot of MOS capacitors under different exposure environments.

field, leakage current density, and $Q_{\mathrm{bd}}$ because of high concentrations of organic compounds.

All these results reveal that AMC will become an important issue in future nanodevice fabrication. An AMC-free manufacturing environment must be seriously considered when manufacturing nanodevices. Advanced ULPA filters, like the NEUROFINE PTFE filter combined with chemical filters, are excellent candidates for creating advanced manufacturing environments.

\section{ACKNOWLEDGMENT}

The authors would like to thank the staff of Daikin Industries, Ltd., Dan-Takuma Technologies Inc., AIR TECH, Ltd., National Nano Device Laboratories, and National Chiao-Tung University Semiconductor Research Center for their analysis and technical support.

\section{REFERENCES}

[1] H. Kitajima and Y. Shiramizu, "Requirements for contamination control in the Gigabit era," IEEE Trans. Semiconduct. Manufact., vol. 10, pp. 267-272, May 1997.

[2] D. Kinkead, M. Joffe, J. Higley, and O. Kishkovich, Forecast of airborne molecular contamination limits for the 0.25 micron high performance logic process, in Technology Transfer \#95 052 812A-TR SEMATECH, May 31, 1995. 
[3] M. Tamaoki, K. Nishiki, A. Shimazaki, Y. Sasaki, and S. Yanagi, "The effect of airborne contaminants in the cleanroom for ULSI manufacturing process," in Proc. 1995 IEEE/SEMI Advanced Semiconductor Manufacturing Conf. Workshop, Nov. 13-15, 1995.

[4] J. K. Higley and M. A. Joffe, "Airborne molecular contamination: Cleanroom control strategies," Solid State Technol., vol. 39, no. 7, pp. 211-212, July 1996.

[5] T. Hattori, "Japanese views on contamination control," Solid State Technol., vol. 37, no. 2, pp. 79-80, Feb. 1994.

[6] M. A. Rappa, "Future directions in controlling particle contamination in semiconductor integrated circuit manufacturing: An industry survey," in Proc. 1994 IEEE/SEMI Advanced Semiconductor Manufacturing Conf. Workshop, New York, NY, Nov. 14-16, 1994.

[7] R. S. Blewer, "Current and future trends in microcontamination research," in Proc. 1994 IEEE/SEMI Advanced Semiconductor Manufacturing Conf. Workshop, New York, NY, Nov. 14-16, 1994.

[8] M. Inoue, T. Noda, M. Togawa, H. Inaba, H. Gomi, T. Yoshida, and T. Okada, "Study on boron contamination in clean room," in Proc. 12th Int. Symp. Contamination Control, Yokohama, Japan, 1994.

[9] T. Jimbo, S. Sakai, K. Katuyama, M. Ito, and H. Tomioka, "Thermal desorption behavior of adsorbed materials on wafer surfaces," in Proc. 1997 IEEE Int. Symp. Semiconductor Manufacturing Conf., San Fancisco, California, Oct. 6-8, 1997.

[10] D. Kinkead and J. Higley, "Targeting gaseous contaminants in wafer fabs: Fugitive amines," Microcontamination, vol. 11, no. 6, pp. 37-40, June 1993.

[11] M. Hashimoto, S. Ichimura, and M. Ide, "Testing a chemically reactive filter for the removal of trace hydrogen sulfide gas," Microcontamination, vol. 12, no. 3, pp. 47-53, Mar. 1994.

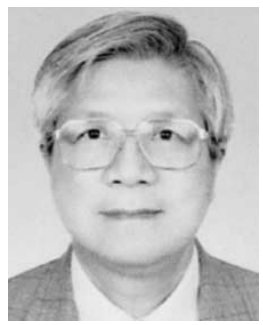

Ching-Fa Yeh was born in Hsinchu, Taiwan, R.O.C. $\mathrm{He}$ received the B.S. degree from the National Oceanic University, Taiwan, in 1971, and the M.S. and $\mathrm{Ph} . \mathrm{D}$. degrees from Tokyo University, Tokyo, Japan, in 1979 and 1982, respectively, all in electrical engineering.

In 1982, he joined Fuji Xerox's Electronic Technology Laboratories, Ebina, where his work has been concerned with research and development of semiconductor process technologies and devices, particularly on dry process technology and contact amorphous-Si image sensor (CIS), and high-voltage devices. Currently, he is a Professor in the Department of Electronics Engineering, National Chiao Tung University, Taiwan. His research interests include poly-Si thin-film devices, power MOSFET devices, liquid-phase deposited (LPD) SiO2 films, ion-plating oxide, and semiconductor wet station.

Dr. Yeh is a member of JJAP.

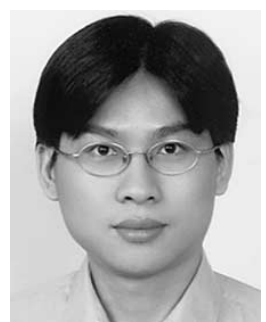

Chih-Wen Hsiao was born in Kaohsiung, Taiwan, R.O.C., on March 20, 1976. He received the B.S. degree in electrical engineering from National Chung-Hsing University, Taichung, Taiwan and the M.S. degree from the Institute of Electronics, National Chiao-Tung University, Hsinchu, Taiwan, in 1998 and 2000, respectively. Since 2000, he has been pursuing the Ph.D. degree at the Institute of Electronics, National Chiao-Tung University.

His current research interests include the key process development of nano-scale semiconductor

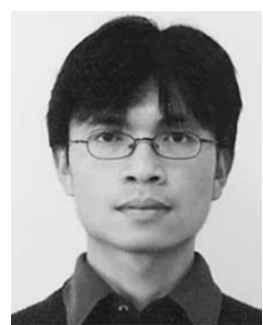

Shiuan-Jeng Lin was born in Taiwan on February 24, 1975. He received the M.S. degree from the Institute of Electronics, National Chiao-Tung University, Hsinchu, Taiwan, R.O.C., in 2001.

Currently, he is with the Taiwan Semiconductor Manufacturing Company.

Chih-Min Hsieh, photograph and biography not available at the time of publication.

Toshio Kusumi, photograph and biography not available at the time of publication.

Hideki Aomi, photograph and biography not available at the time of publication.

Hideo Kaneko, photograph and biography not available at the time of publication.

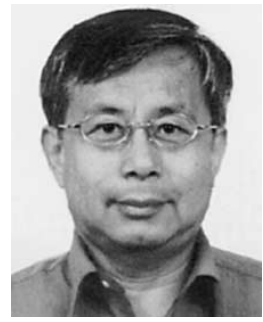

Bau-Tong Dai received the B.S. degree in electrical engineering from Cheng-Kung University in 1968 and the M.S. degree from the Institute of Electronics, National Chiao-Tung University, Hsinchu, Taiwan, R.O.C., in 1970. He received the Ph.D. degree in electrical engineering from the University of Southern California in 1987.

Since 1992, he has been a Researcher with the National Nano Device Laboratories. He has conducted research in chemical-mechanical polishing, copper plating and electropolishing, low $\mathrm{k}$ material, thin film deposition and etching, and MEMS fabrications. He has authored or coauthored over 100 technical papers. His research interests include nanotechnology fabrication, nano science and technology, and VLSI technology. From 1990 to 1992, he was the Vice President of Taiwan Mosel Corp. with the responsibility of wafer foundry, quality control, and production control. He held a variety of positions from process Engineer, Manager, to Director in wafer fabrication and process development, Electronics Research and Service Organization, Industrial Technology Research Institute from 1974 to 1990 . He was one of the key members to build and setup the first wafer fab plant in Taiwan. He also organized the first technology development teams of TFT fabrication, RF technology, CCD fabrication and high-power CMOS technology in ITRI, Taiwan.

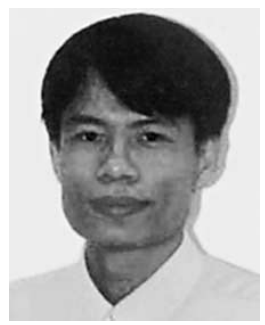

Ming-Shih Tsai was born in Kaohsiung, Taiwan, R.O.C., in 1968. He received the B.S. degree in chemistry from Fu-Jen Catholic University, Taipei, Taiwan in 1990 and the Ph.D. degree in inorganic chemistry from National Taiwan University, Taipei, in 1995.

He joined the National Nano Device Laboratories (NDL) as an Associate Researcher in 1995. He is engaged in developing CMP technology and wet cleaning process on IC manufacturing. His research interests include Oxide CMP, Metal (W, Al alloys, $\mathrm{Cu}$ ) CMP, low dielectric constant SOG CMP, Cu plating, and the Damascene process for multilevel interconnect.

devices. 